\title{
Energy Relaxation at a Hot-Electron Vortex Instability
}

\author{
James M. Knight* and Milind N. Kunchur \\ Department of Physics and Astronomy, University of South Carolina, Columbia, SC 29208
}

(Dated: Recieved on June 28, 2006; published on August 24, 2006)

\begin{abstract}
At high dissipation levels, vortex motion in a superconducting film has been observed to become unstable at a certain critical vortex velocity $v^{*}$. At substrate temperatures substantially below $T_{C}$, the observed behavior can be accounted for by a model in which the electrons reach an elevated temperature relative to the phonons and the substrate. Here we examine the underlying assumptions concerning energy flow and relaxation times in this model. A calculation of the rate of energy transfer from the electron gas to the lattice finds that at the instability, the electronic temperature reaches a very high value close to the critical temperature. Our calculated energy relaxation times are consistent with those deduced from the experiments. We also estimate the phonon mean free path and assess its effect on the flow of energy in the film.
\end{abstract}

PACS numbers: 71.10.Ca, 71.38.-k, 72.10.Di, 72.15.Lh, 73.50.Fq, 74.25.Fy, 74.72.Bk, 74.78.Bz

\section{INTRODUCTION}

When a film of a type II superconductor is placed in a magnetic field large enough to permit penetration of vortices, a transport current in the film acts on the vortices through a Lorentz force that is opposed by a pinning force and, eventually, by a drag force. When the Lorentz force exceeds the pinning force, the vortices are set into motion and the drag force comes into play. When the Lorentz force is substantially larger than the pinning forces but the transport current is still small compared to the depairing current, previous experiments ${ }^{1.2 .3}$ showed that the resulting dissipation is reasonably well described by the Bardeen-Stephen (BS) model ${ }^{4}$. In this region it is Ohmic, but as the current is increased, it becomes nonlinear and eventually reaches an instability manifested by a discontinuous increase in voltage. At temperatures not far below the critical temperature, the instability has been studied in a classic paper ${ }^{5}$ by Larkin and Ovchinnikov (LO). They showed that the electron distribution departs from a thermal distribution at high vortex velocities, changing the superconducting order parameter and altering the drag force on the vortices. They predicted a non-linearity in the current-voltage characteristic and an instability in the vortex motion when the vortices reach a critical velocity $v^{*}$. The LO instability is due to a decrease in the drag force with increasing vortex velocity, accompanied by a decrease in vortex size. LO showed that the critical velocity is independent of the magnetic field. Early experiments on low- $T_{C}$ systems ${ }^{\underline{6}}$ confirmed Larkin and Ovchinnikov's results and predictions. Subsequent experiments on $\mathrm{Y}_{1} \mathrm{Ba}_{2} \mathrm{Cu}_{3} \mathrm{O}_{7-\delta}$ (YBCO) by Doettinger, Huebener, Gerdemann, Kühle, Anders, Träuble, and Villègier ${ }^{\underline{7}}$ and by Xiao and Ziemann ${ }^{\stackrel{8}{ }}$, also confirmed LO behavior.

However, experiments carried out at lower temperatures 9.10 on $\mathrm{YBCO}$, showed a non-linearity and instability with a very different dependence of $v^{*}$ on the magnetic field $B$. Analysis 9.10 showed that the new behavior could be accounted for by a simple model in which the electron gas has a thermal-like distribution function characterized by a higher temperature than the lattice and bath. Larkin and Ovchinnikov did, in fact, suggest this possibility in their original paper ${ }^{11}$ without exploring its consequences. As the electron temperature rises, the resulting increase in resistivity causes a decrease in current above a certain electric field and hence a non-monotonic response. This model yields a critical vortex velocity $v^{*}$ at instability that is proportional to $1 / \sqrt{B}$, as seen in the low- $T$ experiments. Some of the essential consequences of such a hot-electron instability were calculated in our earlier papers and shown to be consistent with experimental observations.

In the present work some of the simplifying assumptions and restrictions in the previous calculations have been removed and more complete calculations have been carried out:

1.) The rate $\tau_{\epsilon}^{-1}$ of transfer of energy from the electron gas to the lattice - which plays a crucial role in determining the electron temperature - was taken as a constant in previous discussions of the model. In this paper we show that it can be expected to have a strong temperature dependence. This temperature dependence of $\tau_{\epsilon}$ is now included in our numerical calculations of the current-voltage curves. We find that the general shape of the current-voltage relation is not very sensitive to the temperature variation of $\tau_{\epsilon}$ because the electron gas passes rapidly from the bath temperature to a temperature not far below $T_{C}$ before any significant non-linearity is manifested. This is a consequence of the very small low-temperature specific heat of a superconducting electron gas. However, the strong temperature variation of the relaxation time gives a sensitive measure of the electron temperature. Evaluation of $\tau_{\epsilon}$ from the data near the instability point indicates an electron temperature much higher than the bath temperature, supporting the heated electron picture of the instability. The calculation of this electron-lattice energy relaxation time is presented in Section III below.

2.) In our previous work ${ }^{9.10}$, we assumed the film thickness to be negligible compared to the phonon mean free path, so that the phonon temperature is uniform 
throughout the film. In this work, we remove this assumption and take phonon lifetime effects into account. The phonons will not necessarily be at the bath temperature, and will have a non-thermal distribution which varies with position in the film. Phonon lifetime effects can be taken into account following work by Bezuglij and Shklovsky ${ }^{13}$, who solved the phonon kinetic equation for a thin film. The non-thermal phonon distribution found in this solution can be incorporated into our calculation of the energy transfer rate, and provides a correction to our earlier results. This result is derived in Section IV below.

We begin in Section II by giving a description of the model presenting some new numerical results for the current-voltage curves under various conditions and for the critical parameters at the instability.

\section{MODEL FOR INSTABILITY}

The macroscopic fields in a type II superconductor carrying a transport current are related to the velocity of the vortices by the fundamental relation

$$
v=\frac{E}{B} c,
$$

which follows from the law of induction. This equation can be used to find the electric field once the vortex velocity is determined by considering the fundamental dissipative processes in the medium. Elastic forces can be shown to be negligible. One of the dissipative processes is the scattering of normal electrons in the vortex core and quasiparticles outside the core first treated by Bardeen and Stephen. They found that the transport current density $j$ is expressed in terms of the upper critical field $H_{c 2}$ and the normal resistivity $\rho_{n}$ by

$$
j=\frac{H_{c 2}}{\rho_{n}} \frac{E}{B} .
$$

Later treatments ${ }^{5.14 .15}$ take into account the relaxation of the order parameter during passage of the vortex first treated by Tinkham ${ }^{12}$. They give results which vary with circumstances, but agree with Eq.(2) to within a numerical factor of order 1 .

These energy dissipation mechanisms raise the energy of the electrons, and this energy subsequently relaxes to the lattice. The assumption of our model is that the electron-electron scattering time is small enough compared to the electron-phonon inelastic scattering time that the electron gas remains in internal thermal equilibrium at a temperature higher than the lattice temperature. The plausibility of the assumption can be checked by estimating the cross-over temperature below which electron-electron scattering is dominant. The standard estimates 16 of the scattering rates $\tau_{e e}^{-1}=\eta \epsilon_{F} / T^{2}$ and of $\tau_{e p}^{-1}=\eta^{3} \omega_{D}^{2} / T^{3}$ then give a cross-over temperature of the order of $100 \mathrm{~K}$ for parameters appropriate to $\mathrm{YBCO}^{25}$.
This temperature is indeed higher than the range of interest in the experiments.

Changes in the energy density of the electron gas can be described by a rate equation that includes the work done by the electric field and the exchange of energy with the lattice. If we assume that the exchange can be described approximately by an energy relaxation time $\tau_{\epsilon}$, then the equation is

$$
\frac{d u}{d t}=j E-\frac{u\left(T^{\prime}\right)-u\left(T_{p}\right)}{\tau_{\epsilon}\left(T^{\prime}, T_{p}\right)},
$$

where $\tau_{\epsilon}$ can depend on the phonon temperature $T_{p}$ as well as on the elevated electron temperature $T^{\prime}$. We argue below that the dependence of $\tau_{\epsilon}$ on $T_{p}$ is weak enough to be ignored in the relevant range of temperatures and the relevant energy transfer rates between the lattice and the bath. The quasiparticles transfer the energy they receive from the transport current to the lattice at a rate much higher than it is radiated back, and the energy then flows from the lattice to the bath. Thus $\tau_{\epsilon}$ can be assumed to depend only on $T^{\prime}$, and we can write the steady-state equation

$$
j E \tau_{\epsilon}=\int_{T_{p}}^{T^{\prime}} c(T) d T
$$

where the energy difference in Eq. (3) has been expressed in terms of the electronic specific heat per unit volume.

Equations (2) - (4) determine the relationship between the electric field, the current density and the temperature. The temperature dependence of the specific heat and the upper critical field are taken from standard BCS theory ${ }^{17}$. In calculating the specific heat, the temperature dependence of the gap was taken from BCS theory and its magnitude was multiplied by a factor to give the observed zero-temperature gap 18 and critical temperature. In the next section, we calculate the energy relaxation time and its temperature dependence.

Typical results of the model are presented in the following figures. Fig. 11 shows the calculated current-voltage curves for different magnetic fields. The shape of the curves is in general qualitative agreement with the experimental data shown in Fig 2. The strength parameter $b$ of the electron-phonon coupling, defined in the next section, was adjusted to obtain agreement with the values of $E$ and $j$ at the peak. We comment on the choice of $b$ in the next section.

The onset of the unstable region in the current-voltage response does not require explicitly invoking the forces on the vortices in treatment of the model. Rather, the instability appears in the result as a region of negative differential conductivity, where $j$ decreases as a function of $E$. The region begins at the value $E^{*}$ of the field that can be determined by calculating $d j / d E$ from Eq.(4), setting the result equal to zero, and solving for $E$ :

$$
E^{*}=\sqrt{\frac{C \rho_{n} B}{\left.H_{c 2} \tau_{\epsilon}^{\prime}+\left(H_{c 2}\right)^{\prime} \tau_{\epsilon}\right)}},
$$


where primes indicate differentiation with respect to temperature 31 The experimentally well-verified $\sqrt{B}$ dependence of $E^{*}$ follows provided the temperature $T^{*}$ at the instability is independent or weakly dependent on $B$ so that the temperature-dependent factors $\mathrm{C}, \tau_{\epsilon}$, and $H_{c 2}$ in Eq. (5. remain independent of $B$. This result is a consequence of our model, since we have explicitly excluded a field dependence for these quantities and taken $\rho_{n}$ to be temperature and field independent. Although Volovik $^{19}$ has shown that the specific heat has a $B$ dependence in type II materials above the lower critical field, we have checked that his scaling prediction at low temperatures gives only a weak dependence in the range of fields $B<<H_{c 2}$ relevant to our experiment. Fig. 3 shows the change in the electron temperature as a function of the applied electric field. The rise in temperature and corresponding decrease in $H_{c 2}$ result in decreasing differential conductivity which leads to the instability. Fig. 4 shows the relatively small effect of increasing the phonon temperature, up to about $40 \mathrm{~K}$, on the final temperature $T^{*}$ reached by the electron gas.

\section{ENERGY TRANSFER RATE}

The total rate at which energy is radiated by the heated quasiparticle gas to the lattice can be calculated by standard methods 20.22 . The two contributing processes, phonon emission and quasiparticle recombination with emission of a phonon, are illustrated schematically in Fig. 5. In the following calculation, applicable to $d$ wave superconductors, we assume the Fermi surface to be a cylinder of radius $k_{F}$ and height $2 \pi / c_{0}, c_{0}$ being the $c$-axis lattice constant. The rate for emission of a phonon of momentum $\mathbf{q}=\mathbf{k}-\mathbf{k}^{\prime}$ by a quasiparticle of momentum

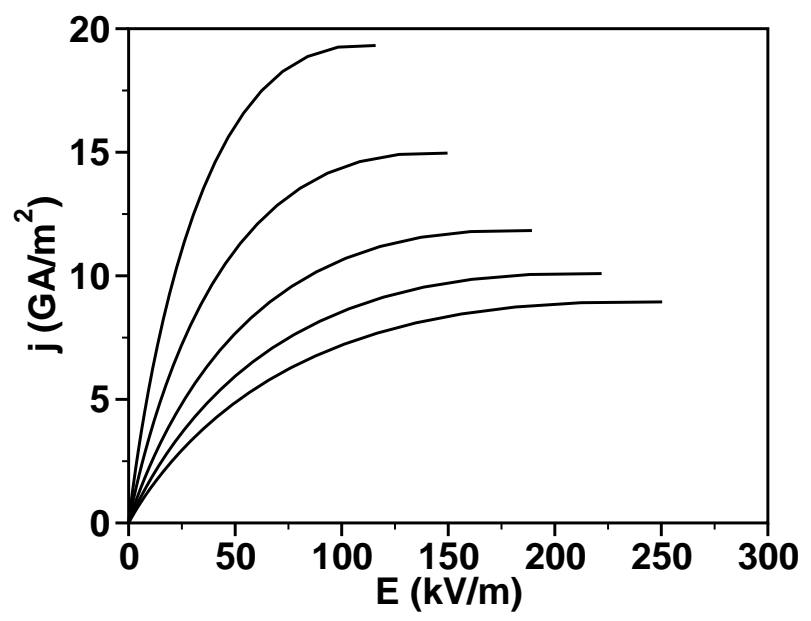

FIG. 1: Effect of increasing flux density on the current density vs electric field curve calculated in the model with variable $\tau_{\epsilon}$. Values of $B$ beginning at the upper curve are $3,5,8,11$, and 14 Tesla.

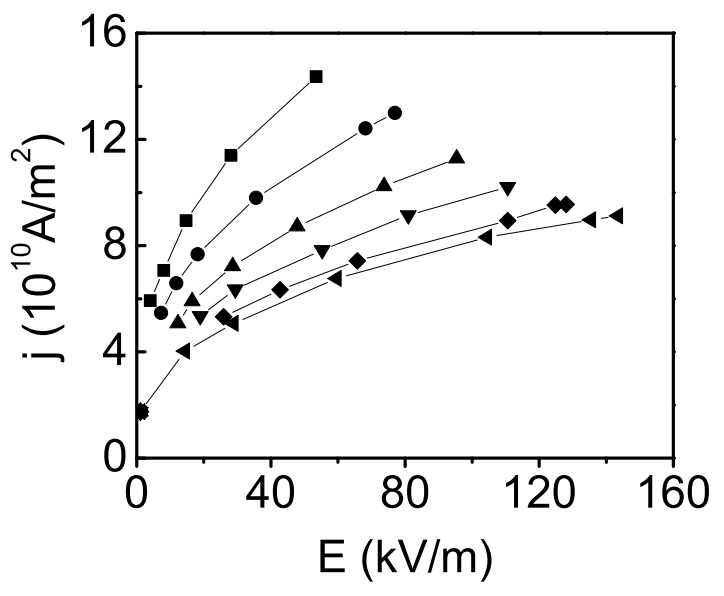

FIG. 2: Experimental curves of current density vs electric field in YBCO for flux density (beginning with the upper curve) $B=3,5,8,11,14$, and 16 Tesla.

$\mathbf{k}$ is

$w=\frac{V}{(2 \pi)^{2} \hbar} \iint d^{3} k^{\prime} d \omega\left|\mathcal{M}_{\mathbf{q}}\right|^{2} \delta\left(\omega-\omega_{\mathbf{q}}\right) \delta\left(E_{\mathbf{k}}-E_{\mathbf{k}^{\prime}}-\hbar \omega\right)$.

This rate can be expressed in terms of the electronphonon spectral function $\alpha^{2}(\omega) F(\omega)$, defined by

$$
\alpha^{2}(\omega) F(\omega)=\frac{V}{(2 \pi)^{3} \hbar^{2}} \int \frac{d S^{\prime}}{v_{F}^{\prime}}\left|\mathcal{M}_{\mathbf{k}-\mathbf{k}^{\prime}}\right|^{2} \delta\left(\omega-\omega_{\mathbf{k}-\mathbf{k}^{\prime}}\right),
$$

where $d S^{\prime}$ is an area element on the Fermi surface and $v_{F}^{\prime}$ is the Fermi velocity. If the quantity $\left|\mathcal{M}_{\mathbf{k}-\mathbf{k}^{\prime}}\right|^{2} \delta(\omega-$

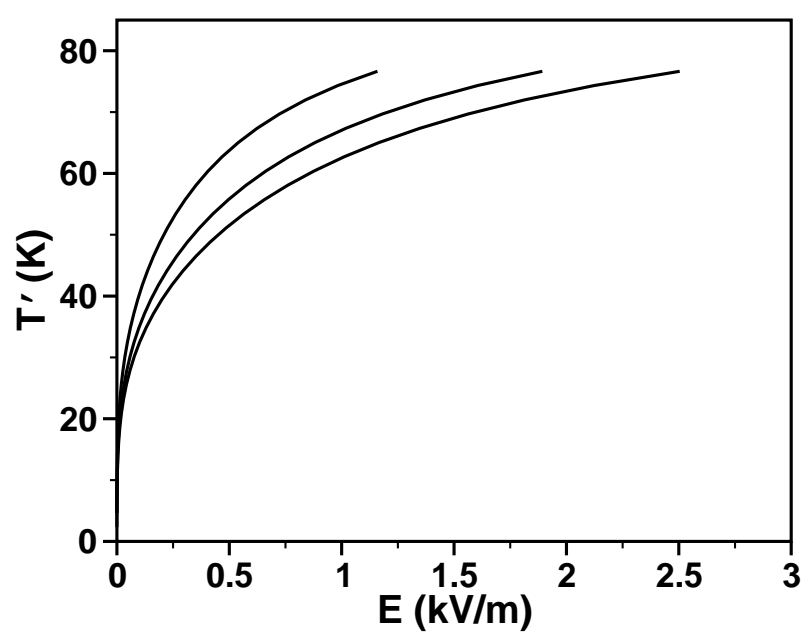

FIG. 3: Calculated electron temperature $T^{\prime}$ vs electric field $E$ for $B=3,8$, and14 Tesla, with $B$ increasing from left to right. 
$\left.\omega_{\mathbf{k}-\mathbf{k}^{\prime}}\right)$ is replaced by its average over the Fermi surface,

$$
\frac{1}{S} \int d S^{\prime}\left|\mathcal{M}_{\mathbf{k}-\mathbf{k}^{\prime}}\right|^{2} \delta\left(\omega-\omega_{\mathbf{k}-\mathbf{k}^{\prime}}\right)=\frac{2 \pi c_{0} \hbar^{2} v_{F}}{V k_{F}} \alpha^{2} F(\omega),
$$

the transition rate becomes

$$
\left.w=\frac{\pi}{3 c_{0} \hbar \gamma} \int d^{2} k^{\prime} \alpha^{2} F\left(E_{\mathbf{k}}-E_{\mathbf{k}^{\prime}}\right) / \hbar\right),
$$

where $d^{2} k^{\prime}=k^{\prime} d k^{\prime} d \theta^{\prime}$ and $\theta^{\prime}$ is the azimuthal angle o the Fermi cylinder. The total energy transfer rate wa obtained by integrating $w$ over initial quasiparticle ener gies, and reduces to

$$
\left.P_{e}=\frac{V}{12 \pi c_{0}^{2} \hbar \gamma} \int d^{2} k \int d^{2} k^{\prime} \alpha^{2} F\left(E-E^{\prime}\right) / \hbar\right)\left(E-E^{\prime}\right) g_{e}(I
$$

The factor $g_{e}\left(E, E^{\prime}\right)$ contains the occupation factors an coherence factors

$$
g_{e}\left(E, E^{\prime}\right)=f(E)\left(1-f\left(E^{\prime}\right)\right)\left(\left(1-\frac{\Delta \Delta^{\prime} \cos 2 \theta \cos 2 \theta^{\prime}}{E E^{\prime}}\right)\right.
$$

for the initial and final states. The second term in the coherence factor integrates to zero because of the $d$-wave symmetry of the order parameter. After transforming the momentum integrals into integrals over the quasiparticle energy and performing the azimuthal integrals, the energy transfer rate reduces to

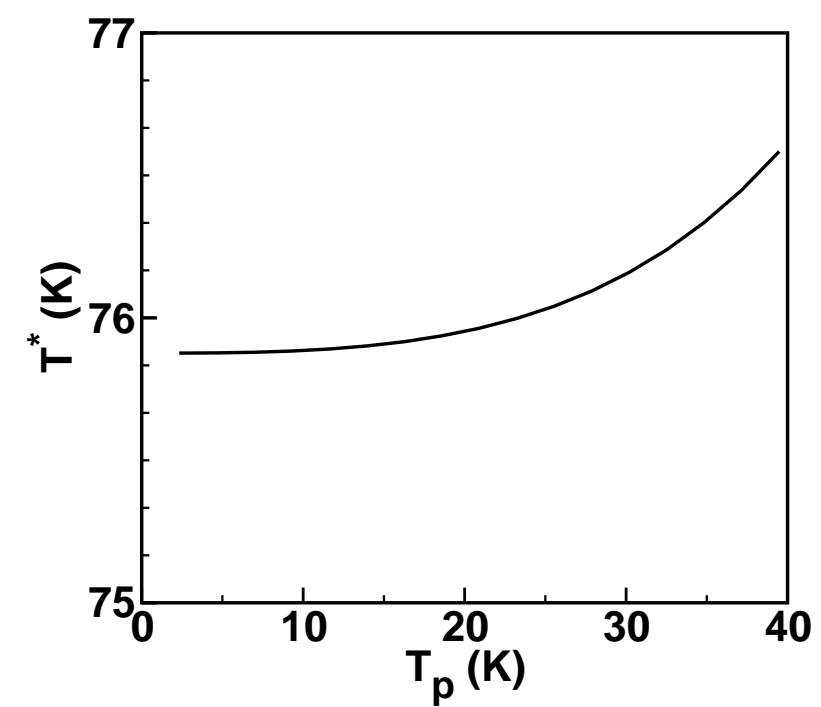

FIG. 4: Electron temperature at the instability vs. phonon temperature

$$
P_{e}=\frac{3 V \gamma}{4 \pi^{3} \hbar} \int E d E D(E, \Delta) \int E^{\prime} d E^{\prime} D\left(E^{\prime}, \Delta\right) F\left(\left(E-E^{\prime}\right) / \hbar\right)\left(E-E^{\prime}\right) g_{e}\left(E, E^{\prime}\right) .
$$

Here $D(E, \Delta)$ is the $d$-wave density of states

$$
\int \frac{d \theta}{\sqrt{E^{2}-\Delta^{2} \cos ^{2} 2 \theta}}= \begin{cases}\frac{4}{\Delta} K\left(\frac{E^{2}}{\Delta^{2}}\right), & E<\Delta \\ \frac{4}{E} K\left(\frac{\Delta^{2}}{E^{2}}\right), & E>\Delta,\end{cases}
$$

where $K$ denotes the elliptic function of the first kind. In this way of calculating, there is some averaging over the Fermi surface, but the characteristic $d$-wave density of states with its logarithmic singularity at $E=\Delta$ has been retained.

The spectral function $\alpha^{2} F(\omega)$ is assumed to be of the form $b \omega^{2}$ appropriate for acoustic phonons. Although optical phonons are present in high temperature superconductors, they are assumed to make only a negligible contribution to the thermal conductivity responsible for carrying energy from the heated electrons to the bath. The spectral function is cut off at the Debye frequency $\omega_{D}$. The constant $b$ measures the strength of the electronphonon interaction. This is usually expressed through the electron-phonon coupling constant $\lambda$, defined as the integral of $2 \alpha^{2} F(\omega) / \omega$ over frequency, which has a value of order unity in most superconductors. Since we are only considering acoustic phonons, this provides an upper limit for $b$ of about $10^{-3} \mathrm{meV}^{-2}$. This is consistent
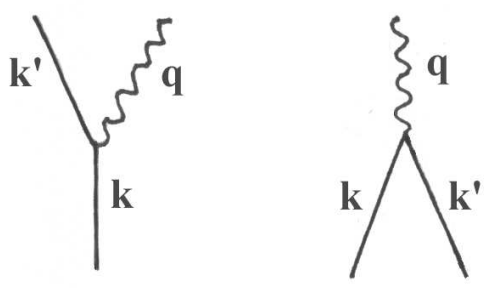

FIG. 5: Diagrams contributing to the energy transfer rate from the quasiparticle gas to the lattice.

with the magnitude of the electron-phonon matrix element quoted in Ref. [21], which gives $b \approx 6 \times 10^{-4}$. It is also in the same range as the values extracted from neutron scattering data by Kaplan et $a l^{20}$ for low temperature superconductors. The deformation potential approximation as cited in Ref. [22], on the other hand, gives a value of the order of $3 \times 10^{-7} \mathrm{meV}^{-2}$. The choice that gives the best peak values for the peak current and for $E^{*}$ is $5.2 \times 10^{-6} \mathrm{meV}^{-2}$, more in line with the latter value. The curves shown have taken this best fit value for $b$. 
Introducing the dimensionless variables $x=E / T, y=$ $E^{\prime} / T$, and $z=\Delta / T$, the energy transfer rate takes the form

$$
P_{e}=G V T^{5} \Phi_{e}
$$

with

$$
G=\frac{3 \gamma b}{4 \pi^{3} \hbar^{3}}
$$

and the dimensionless integral $\Phi_{e}$ given by

$$
\Phi_{e}=\int_{0}^{\infty} d x \int_{0}^{x} \frac{D(x, z) D(y, z) x y(x-y)^{3}}{e^{x}+e^{x-y}+e^{-y}+1} .
$$

$D(x, y)$ is the dimensionless form of the density of states Eq. [13. The quasiparticle recombination process gives an expression of the same form with $\Phi_{e}$ replaced by

$$
\Phi_{r}=\int_{0}^{d} d x \int_{0}^{d-x} d y \frac{D(x, z) D(y, z) x y(x+y)^{3}}{e^{x}+e^{x+y}+e^{y}+1} .
$$

The value of the specific heat constant $\gamma$ is taken from measurements of W. C. Lee, et. al ${ }^{23}$ as $0.0331 \mathrm{meV}^{-1}$ $\mathrm{nm}^{-3}$, giving $G=1.257 \times 10^{6} \mathrm{meV}^{-1} \mathrm{~nm}^{-3} \mathrm{~s}^{-1}$ when $b$ is normalized to give $\lambda=1$.

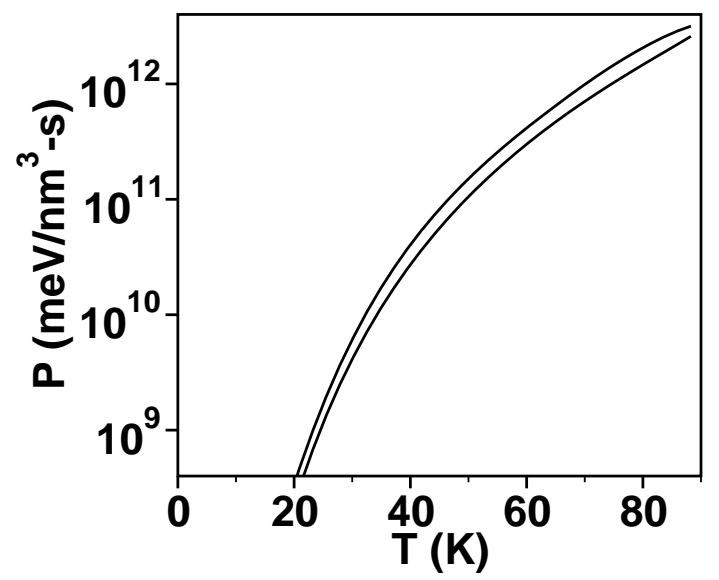

FIG. 6: Calculated energy transfer rate in $\mathrm{meV} / \mathrm{nm}^{3}$-s of electron gas at temperature $T$ to a zero temperature lattice. The lower curve represents the phonon emission process and the upper curve represents the quasiparticle annihilation process.
Fig. 6] shows the calculated energy transfer rates for emission and recombination. Fig. [7] shows the energy relaxation time $\tau_{\epsilon}$ found by equating the total transfer rate from both processes to the last term in the rate equation (Eq. 3).

We note the following properties of the energy transfer rate:

1.) The rate of energy transfer from the electrons to the lattice at any given temperature is equal to the rate of transfer from the lattice to the electrons at the same temperature. Indeed, the rate from phonon emission balances the rate from absorbtion and the rate from

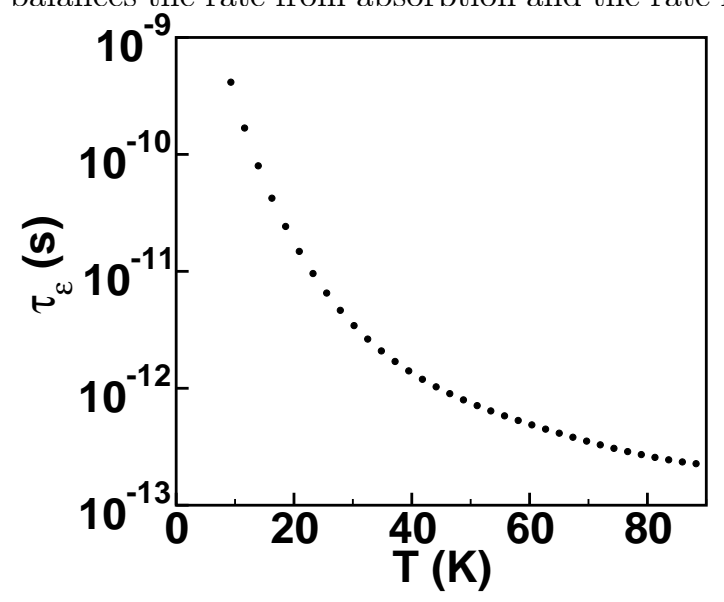

FIG. 7: Energy relaxation time $\tau_{\epsilon}$ as a function of temperature.

quasiparticle recombination balances the rate from pair creation. These results can be demonstrated in the deformation potential approximation, where the matrix element for phonon emission and absorption depends only on the phonon energy $\nu$. For example, the rates for emission and absorption and for quasiparticle recombination and creation can be written

$$
\begin{aligned}
& P_{e}=G \int(n(\nu)+1) f_{e}(E, E-\nu) N(E) N(\nu-E) \nu^{3} d E d \nu \\
& P_{a}=G \int n(\nu) f_{a}(E, E+\nu) N(E) N(E+\nu) \nu^{3} d E d \nu \\
& P_{r}=G \int(n(\nu)+1) f_{r}(E, \nu-E) N(E) N(\nu-E) \nu^{3} d E d \nu \\
& P_{c}=G \int n(\nu) f_{c}(E, \nu-E) N(E) N(\nu-E) \nu^{3} d E d \nu
\end{aligned}
$$


where $n(\nu)$ is the phonon occupation number at the given temperature and $N(E)$ is the quasiparticle density of states, and $G$ has the value $3 V b \gamma / 4 \pi^{3} \hbar^{3}$. The equality of the rates is evident upon substituting the explicit forms of the Fermi and Bose distribution functions. In the same manner, the emission and absorption rates are identical after the same substitutions and the change of variable $E^{\prime}=E+\nu$ in $P_{a}$.

2.) The differences between the emission and absorption rates and between the pair recombination and creation rates have only a weak dependence on the lattice temperature as long as the electron temperature is near $T_{c}$ and the lattice temperature is low, say $T_{p} \leq T_{c} / 2$. This conclusion is based on values for $T_{c}(7.75 \mathrm{meV})$ and $\Delta(19 \mathrm{meV})$ for YBCO. Wellstood, Urbina, and Clarke $\stackrel{22}{,}$, assert that the difference between the emission rate and the absorption rate for a normal metal is equal to the difference between the rate electrons radiate to a zero temperature lattice and rate phonons radiate to a zero temperature electron gas. This result is only approximately valid in the gas of quasiparticles. The differences can be calculated from the previous pairs of equations by taking $n(\nu)$ to be the phonon distribution function at $T_{p}$. The difference between emission and absorption rates, for example, is:

$P_{e}-P_{a}=G \int_{0}^{\infty} d E \int_{0}^{E-\Delta} d \nu f_{e}(E, E-\nu) \frac{e^{\nu / T_{p}}-e^{\nu / T^{\prime}}}{e^{\nu / T_{p}}-1}$.

The dependence on $T_{p}$ is contained in the last factor. For $\nu$ of the order of $\Delta, T^{\prime}$ of the order $T_{c}$, and $T_{0}$ in the range zero to $T_{c} / 2$, this factor only varies from 1.0 to 0.915 , showing therefore a weak dependence of the difference on $T_{p}$. In an earlier calculation assuming $s$ wave symmetry of the order parameter, the differences were calculated explicitly for $T=0.8 T_{c}$ and $T_{p}$ ranging from $0.1 T_{c}$ to $0.5 T_{c}$. The difference varies less than $10 \%$ for emission and absorption and less than $1 \%$ for the dominant creation and recombination. In view of these results, we ignore the dependence on $T_{p}$ and calculate $\tau_{\epsilon}$ on the basis of the radiation rate to a zero temperature lattice. A very similar argument applies to the difference between the pair recombination and pair creation rates.

3.) Quasiparticle emission and absorbtion can only satisfy the energy and momentum conservation laws if the the quasiparticle velocity $v_{F} \partial E / \partial \epsilon$ before emission or after absorbtion is greater than the sound velocity. This Cerenkov condition should be taken into account in the averaging near the Fermi surface that enters into calculation of the electron-phonon spectral function $\alpha^{2} F$. In the integrals over quasiparticle energy above, the lower limit should be the energy $E_{c}$ at which quasiparticles reach the sound velocity rather than 0 . The correction is of the order of the square of the ratio of the sound velocity to the Fermi velocity. Since $s / v_{F}<<1$ for all superconductors $\left(s / v_{F} \approx 1.5 \times 10^{-2}\right.$ in YBCO $)$, the correction can be safely ignored.

\section{PHONON LIFETIME EFFECTS}

In the above discussion, we have not distinguished the phonon temperature and the bath temperature. We now consider corrections arising from a more general treatment of the phonon distribution. The standard estimate of phonon mean free path for normal metals $\hbar v_{F} / k T$ gives $17 \mathrm{~nm}$ when the Fermi velocity is taken to be $2 \times 10^{5}$ $\mathrm{m} / \mathrm{s}$, which is shorter than the $100 \mathrm{~nm}$ thickness of the experimental films. The estimate of Kaplan, Chu, Langenberg, Chang, Jafarey, and Scalapino 20 of quasiparticle and phonon lifetimes in an $s$-wave superconductor below the critical temperature gives a frequency- and temperature-dependent numerical factor of order unity multiplied by the characteristic time

$$
\tau_{0}^{p h}=\frac{\hbar N\left\langle\alpha^{2}\right\rangle_{a v}}{4 \pi^{2} N(0)} \Delta(0),
$$

where $N$ is the ion number density, $\left\langle\alpha^{2}\right\rangle_{a v}$ is the average electron-phonon coupling constant, $N(0)$ is the singlespin electronic density of states at the Fermi surface, and $\Delta(0)$ is the zero-temperature gap. Taking 25 the values $N=13$ per unit cell, $\left\langle\alpha^{2}\right\rangle_{a v}=5 \mathrm{meV}, N(0)$ calculated from the free-electron theory with $v_{F}$ having the value quoted above, and $\Delta(0)=19 \mathrm{meV}$, and converting the lifetime to a mean free path using the longitudinal sound velocity $4.2 \times 10^{3} \mathrm{~m} / \mathrm{s}$ yields a path of the order of $10^{3}$ $\mathrm{nm}$, an order of magnitude larger than the thickness of the experimental film.

These estimates indicate that we are dealing with a case in which the phonon mean free path could be comparable to the thickness of the sample. To deal with the general case, we follow Bezuglij and Shklovskij ${ }^{13}$, writing the kinetic equation for the phonon distribution function $n(\mathbf{q}, z)$ as

$$
s_{z} \frac{\partial n(\mathbf{q}, z)}{\partial z}=-\frac{n(\mathbf{q}, z)-n(T)}{\tau_{p h}},
$$

where $s_{z}$ is the component of the sound velocity perpendicular to the plane of the film and $n(T)$ is the thermal phonon distribution at the electron temperature. If phonons are reflected at the free surface of the film and transmitted with average coefficient $\alpha$ at the substrate interface, it is found that the phonon distribution function is a linear combination of two thermal distributions, one at the bath temperature and one at the electron temperature. The coefficients in the linear combination depend on the position within the film and on the direction of propagation of the phonons:

$$
n=A(z, \theta) n(T)+B(z, \theta) n\left(T_{0}\right) .
$$

The integrand in the expression for the energy transfer rate from quasiparticle gas to lattice contains the factor $n+1$, while that for the reverse rate contains a factor $n$. If Eq. (20) is substituted into these rates and account is taken of the condition for equilibrium between 
the lattice and the gas, the resulting rate contains a term with the factor $1-A$ and a term with the factor $B n\left(T_{0}\right)$. For purposes of estimating the correction for finite phonon lifetime, we neglect the term proportional to $n\left(T_{0}\right)$ compared to the $1-A$ term on the ground that the phonon number is small at a temperature $T_{0}$ which is much smaller than the Debye temperature. An estimate of the remaining term can be obtained by replacing $1-A$ in the integral for the rate by its average value over the thickness of the film and over the directions of propagation of the phonon. The remaining integral is the one we evaluated in the previous section.

The resulting explicit expression for $A$ is:

$$
A=1-\frac{\alpha}{1-(1-\alpha) e^{-2 d / l_{z}}}\left\{\begin{array}{ll}
e^{-z / l_{z}} & , q_{z}>0 \\
e^{-(2 d-z) / l_{z}} & , q_{z}<0
\end{array}\right\} .
$$

Eq. 20] with 21 reflects the gradual change of the distribution from a nearly thermal distribution at the bath temperature at the substrate interface $z=0$ to an electron temperature thermal distribution over the distance of a phonon mean free path. The transmission probability $\alpha$ can be determined in principle ${ }^{26}$ from the measured value of the thermal resistance of the film-substrate interface, defined as the ratio of $\Delta T$ at the interface to the product of the power dissipated per unit volume and the thickness of the film. The measured value ${ }^{27.28}$ for YBCO is about $1 \times 10^{-3} \mathrm{Kcm}^{2} / \mathrm{W}$. The determination of $\alpha$ is affected by uncertainties due to the averaging and due to the temperature variation of the thermal resistance. A literal application of Eq.[14] of Ref. 26] produces the average value 0.184 when $d$ is of the order of or larger than $l$. When $d \ll l$, Shklovskii shows that the effective $\alpha$ is $2 d / l$, which is 0.2 for the longest estimate of phonon mean free path above. We therefore accept 0.2 as a reasonable value. Sensitivity of the value of $1-A$ to $d / l$ and $\alpha$ are shown in Table $\llbracket$ The longest estimate of phonon mean free path with the best estimate of the transmission coefficient indicate that the energy transfer rate will be multiplied by a factor of 0.325 due to phonon lifetime effects.

\begin{tabular}{|c|c|c|c|}
\hline$d / l$ & $\alpha=0.8$ & $\alpha=0.5$ & $\alpha=0.2$ \\
\hline 6.0 & 0.0333 & 0.0208 & 0.00833 \\
\hline 1.0 & 0.190 & 0.121 & 0.0493 \\
\hline 0.1 & 0.680 & 0.553 & 0.325 \\
\hline
\end{tabular}

TABLE I: Calculated values of the phonon lifetime factor $1-A$ for three values of the ratio of thickness $d$ to phonon mean free path $l$ and three values of the average transmission coefficient $\alpha$.

\section{CONCLUSION}

At temperatures well below $T_{C}$, high electric fields and current densities can produce an instability that can be accounted for by a hot electron gas model in which the electronic temperature is elevated due to dissipation. The calculations presented here provide a quantitative justification for this scenario by showing that the temperature variation of the energy transfer rate between the lattice and the electrons is consistent with the position of the instability observed in YBCO films. They show also that if the phonon mean free path is not too small compared to the film thickness, the necessary temperature difference between electrons and lattice can be maintained.

\section{ACKNOWLEDGEMENTS}

We thank B. I. Ivlev for important suggestions, and M. Geller, R. P. Huebener, and R. P. Prozorov for helpful discussions and criticisms. This work was supported by the U. S. Department of Energy through grant number DE-FG02-99ER45763.
* Electronic address: knight@sc.edu

$\dagger$ Electronic address: kunchur@sc.edu URL: http://www .physics.sc.edu/kunchur

1 M. N. Kunchur, D. K. Christen, and J. M. Phillips, Phys. Rev. Lett. 70, 998 (1993).

2 M. N. Kunchur, D. K. Christen, C. E. Klabunde, and J. M. Phillips, Phys. Rev. Lett. 72, 2259 (1994).

3 M. N. Kunchur, B. I. Ivlev, D. K. Christen, and J. M. Phillips, Phys. Rev. Lett. 84, 5204 (2000).

4 J. Bardeen and M. J. Stephen, Phys. Rev. 140, A1197 (1965).

5 A. I. Larkin and Yu. N. Ovchinnikov, in Nonequilibrium Superconductivity, D. N. Langenberg and A. I. Larkin, eds. (Elsevier, Amsterdam 1986), Chapter 11.

6 W. Klein, R. P. Huebener, S. Gauss, and J. Parisi, J. Low Temp. Phys. 61, 413 (1985); and L. E. Musienko, I. M. Dmitrenko, and V. G. Volotskaya, Pis'ma Zh. Eksp.Teor.
Fiz. 31, 603 (1980) [JETP Lett. 31, 567 (1980)].

7 S. G. Doettinger, R. P. Huebener, R. Gerdemann, A. Kühle, S. Anders, T. G. Träuble, and J. C. Villègier, Phys. Rev. Lett. 73, 1691 (1994).

8 Z. L. Xiao and P. Ziemann, Phys. Rev. B 53, 15265, (1996).

9 M. N. Kunchur, Phys. Rev. Lett. 89, 137005 (2002).

10 M. N. Kunchur and J. M. Knight, Mod. Phys. Lett. B 17, 549 (2003).

11 A. I. Larkin and Yu. N. Ovchinnikov, Zh. Eksp. Teor. Fiz. 68, 1915 (1975)[Sov Phys. JETP 41, 960 (1976)].

12 M. Tinkham, Phys. Rev. Lett. 13, 804 (1964).

13 A. I. Bezuglij and V. A. Shklovskij, Physica C, 202, 234 (1992).

14 C. R. Hu and R. S. Thompson, Phys. Rev. B6, 110 (1972); and R. S. Thompson and C. R. Hu, Phys. Rev. Lett. 27, 1352 (1971).

15 N. P. Kopnin, Theory of Nonequilibrium Superconductivity 
(Oxford University Press, Oxford 2001), Chapter 12.

16 A. Abrikosov, Fundamentals of the Theory of Metals (North Holland, Amsterdam 1988).

17 N. R. Werthamer, E. Helfand, and P. C. Hohenberg, Phys. Rev 147, 295 (1966).

18 Wang Lanping, He Jian, and Wang Guowen., Phys. Rev. B 40, 10954 (1989); and R. T. Collins, Z. Schlesinger, R. H. Koch, R. B. Laibowitz, T. S. Plaskett, P. Freitas, W. J. Gallagher, R. L. Sandstrom, and T. R. Dinger, Phys. Rev. Lett. 59, 704 (1987).

19 G. E. Volovik, Pis'ma Zh. Eksp. Teor. Fiz. 65, 465 (1997) [JETP Lett. 65, 491 (1997)].

20 S. B. Kaplan, C. C. Chu, D. N.Langenberg, J. J. Chang, S. Jafarey,and D. J. Scalapino, Phys. Rev. B 14, 4854 (1976).

${ }^{21}$ Neil W. Ashcroft and N. David Mermin, Solid State Physics (Sanders College Publishers, Fort Worth, 1976).

22 F. C. Wellstood, C. Urbina, and John Clarke, Phys. Rev. B 49, 5942 (1994).

23 W. C. Lee, K. Sun, L. L. Miller, D. C. Johnston, R. A. Klemm, S. Kim, R. A. Fisher, and N. E. Phillips, Phys.
Rev. B 43, 463 (1991).

24 J. M. Ziman, Principles of the Theory of Solids (Cambridge University Press, Cambridge, 1979).

25 NIST WebHTS Database, http://www.ceramics.nist.gov/srd/hts/ htsquery.htm

${ }^{26}$ V. A. Shklovskii, Zh. Eksp. Teor. Fiz. 78, 1281 (1980) [Sov. Phys. JETP 51, 646 (1980)].

27 M. Nahum, S. Verghese, P. L. Richards, and K. Char, Appl. Phys. Lett. 59, 2034 (1991).

28 M. L. Kunchur, Mod. Phys. Lett. B 9, 299 (1995).

29 M. N. Kunchur, B.I. Ivlev, and J. M. Knight, Phys. Rev. Lett. 87, 177001 (2001).

30 M. N. Kunchur, B.I. Ivlev, and J. M. Knight, Phys. Rev. B 66, 060505 (2002).

31 A secondary consequence of this instability is a fragmentation of the flux flow in the region of negative $d j / d E$ resulting in steps in the current-voltage curves. Such steps have been observed in constant-voltage measurements at high dissipations ${ }^{29,30}$. 